\title{
Abelian dominance and adjoint color sources
}

\author{
Grigorios I. Poulis ${ }^{\mathrm{a} *}$ \\ a National Institute for Nuclear Physics and High Energy Physics (NIKHEF) \\ P.O. Box 41882, 1009 DB Amsterdam, The Netherlands
}

\begin{abstract}
Abelian dominance in the case of color sources in the fundamental representation is shown to follow from certain properties of maximal abelian projected $\mathrm{SU}(2)$ gauge theory. The possibility of having an analog of abelian dominance in the case of adjoint representation sources is addressed in the same framework.
\end{abstract}

\section{Maximal Abelian Projection revisited}

In the abelian projetion (AP) theory of confinement [1] partial gauge-fixing projects onto the $\mathrm{U}(1)^{\mathrm{N}-1}$ Cartan subgroup of the original $\mathrm{SU}(\mathrm{N})$ gauge symmetry (henceforth we take $\mathrm{N}=2$ ). With respect to this residual abelian symmetry, diagonal gluons transform as "photons", while offdiagonal gluons are doubly-charged matter fields. Quark fields in the fundamental $(j=1 / 2)$ representation are singly-charged $(n=1)$, while quarks in the adjoint $(j=1)$ representation are neutral when $m_{j}=0$ and doubly-charged $(n=2)$ when $m_{j}= \pm 1$. The maximal abelian (MA) projection [2], corresponding to maximizing

$$
\sum_{x, \mu} \operatorname{Tr}\left[\sigma_{3} U_{x, \mu} \sigma_{3} U_{x, \mu}^{\dagger}\right]=\sum_{x, \mu} \cos \left(2 \phi_{x, \mu}\right)
$$

has provided some evidence [3, 4 in support of this scenario of confinement. Having set $U_{11}=$ $\cos \phi e^{i \theta}, U_{12}=\sin \phi e^{i \chi}$, etc., the $\mathrm{SU}(2)$ plaquette action $S_{P}$ can be decomposed as $S_{P}=S_{\theta}+$ $S_{\chi}+S_{\theta \chi}[5]$, the subscripts denoting which of the $\theta, \chi$ fields appear, e.g., $S_{\theta}=\left(\prod_{i \in \square} \cos \phi_{i}\right) \cos \theta_{\square}$, where $\theta_{\square}$ is a $\mathrm{U}(1)$-invariant abelian plaquette. $S_{\theta}$ and $S_{\chi}$ have one term each, while $S_{\theta \chi}$ has six. Due to the gauge fixing condition, Eq. (1), $S_{\chi}<S_{\theta \chi}<S_{\theta}$. Using 50 configurations on a $12^{4}$ lattice at $\beta=2.4$ we find $\langle\cos \phi\rangle \approx 2 / 3$ (the random value) in local gauges [7]. In MA projection $\langle\cos \phi\rangle$ is close to 1 , and, accordingly, $S_{\theta}$ dominates the action, i.e., $\left\langle S_{P}\right\rangle=\left\langle S_{\theta}\right\rangle$ within $9 \%$. Furthermore, we find that $\cos \phi$ behaves more

\footnotetext{
*Research supported by Human Capital and Mobility Grant ERBCHBICT941430.
}

like a parameteter than a dynamical variable and factorizes in expectation values, e.g., $\left\langle\prod_{\square} \cos \phi\right\rangle$ $=\langle\cos \phi\rangle^{4}$ and $\left\langle S_{\theta}\right\rangle=\left\langle\prod_{\square} \cos \phi\right\rangle\left\langle\cos \theta_{\square}\right\rangle$ within $0.2 \%$ and $3 \%$, respectively. These results suggest that $\chi$ fields can be treated as beeing essentially random, and MAQCD, the effective abelian theory after maximal abelian projection, is basically compact QED with effective coupling $\beta\langle\cos \phi\rangle^{4}$.

\section{Abelian Dominace}

\subsection{Operational Definition}

A interesting feature of MA projection, named "abelian dominance", is that abelian Wilson loops $W_{n=1}=\cos \left(\sum_{i \in L} \theta_{i}\right)$ reproduce the fundamental $\mathrm{SU}(2)$ string tension [6]. Abelian dominance is essentially an empirical observation. Operationally, one may define abelian dominance $(\mathrm{AD})$ as the property that large-scale properties of QCD are reproduced by operators $W_{\text {abel }}$, constructed exclusively from the abelian phases $\theta[3]$. We distinguish between two versions:

- strong version: the operators $W_{\text {abel }}$ are obtained by using (after MA projection) rescaled, diagonal links, $U=\operatorname{diag}\left[e^{i \theta}, e^{-i \theta}\right]$, in the place of full $\mathrm{SU}(2)$ links.

- weak version: a suitable Ansatz must be devised for constructing the abelian operators.

Clearly, if the strong version is satisfied, so is the weak, but not vice versa.

\subsection{Fundamental Sources}

For an $T \times R$ Wilson loop in the fundamental representation, $W_{j=1 / 2}=w_{0}+i \vec{\sigma} \cdot \vec{w}$, we can write 
a similar decomposition as for the plaquette [7]

$$
\begin{gathered}
w_{0}+i w_{3}=(\cos \phi)^{2 L} e^{i \theta_{L}}+(\sin \phi)^{2 L} e^{i \chi_{L}} \\
+\sum_{m=1}^{L-1}(\cos \phi)^{2 m}(\sin \phi)^{2(L-m)} \sum_{n=1}^{\left(\begin{array}{c}
2 L \\
2 m
\end{array}\right)} s_{n} e^{i \Omega_{n}[\theta, \chi]} .
\end{gathered}
$$

Consider now $\left\langle W_{j=1 / 2}\right\rangle=\left\langle w_{0}\right\rangle$. Carrying out the free- $\chi$ intregration as remarked in Sec. I, we find

$$
\left\langle W_{j=1 / 2}\right\rangle \approx\langle\cos \phi\rangle^{2 L}\left\langle\cos \theta_{L}\right\rangle \text {. }
$$

Since the two expectation values differ by a perimeter $(L \equiv T+R)$ term only, they generate the same string tension. Thus, according to the weak version of abelian dominance, the abelian operator $W_{\text {abel }}^{1 / 2}$ in this case should be the singlycharged abelian Wilson loop, $W_{n=1}=\cos \theta_{L}$. Indeed, from Fig. 1 one sees that Eq. (3) is a

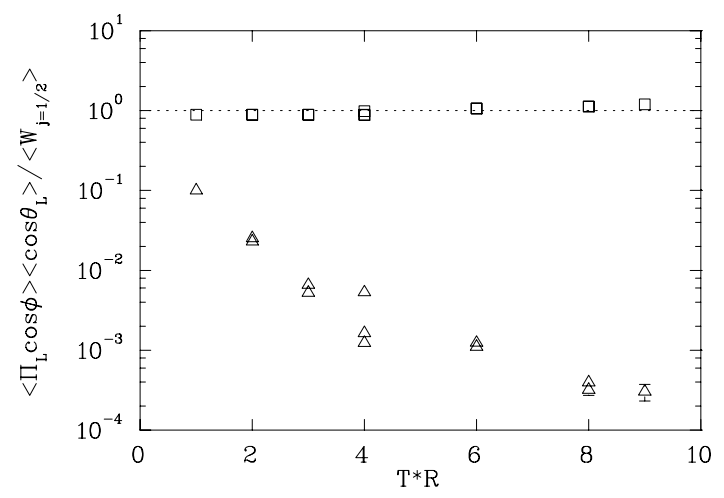

Figure 1. Testing approximation Eq. (3) in F12 $(\triangle)$ and MA $(\square)$ projection.

good approximation in MA projection and, not surprisingly, very bad in F12 projection. Notice that $\cos \theta_{L} \equiv \cos \left(\sum_{i \in L} \theta_{i}\right)$ can also be thought of as a fundamental-representation Wilson loop constructed from rescaled, diagonal SU(2) links $W_{j=1 / 2}^{d}=\operatorname{Tr}\left(\prod_{i \in L} \operatorname{diag}\left[e^{i \theta_{i}}, e^{-i \theta_{i}}\right]\right)$. Thus, with respect to $\mathrm{AD}$ for fundamental repr. sources

1. the difference between $\cos \phi \simeq 1$ and $\cos \phi$ $=1$ is not essential, i.e., MAQCD can be regarded as a "diagonal $\mathrm{SU}(2)$ " theory.

2. there is no distinction between weak and strong versions; both are satisfied.

\subsection{Adjoint Sources}

Abelian dominance for adjoint sources (quarks) requires that the adjoint "string tension" $\sigma_{j=1}$ can be extracted from some abelian correlator $W_{\text {abel }}^{1}$. For the strong version of $\mathrm{AD}$, this operator is the adjoint Wilson loop from diagonal $\mathrm{SU}(2)$ links [8,7]

$W_{j=1}^{d}=\frac{2}{3} \cos \left(2 \theta_{L}\right)+\frac{1}{3}$.

As realized by Greensite and coworkers 8.9.9], the persistence of Casimir scaling $\left(\sigma_{j=1} \approx \frac{8}{3} \sigma_{j=1 / 2}\right)$ in $\mathrm{MC}$ simulations, and the associated failure to unambigously verify screening for the potential between adjoint sources ([9] and references therein) presents a challenge for abelian dominance: if MAQCD is a "diagonal $\mathrm{SU}(2)$ " theory, there is no way for two $m_{j}=0$ (neutral) components of the adjoint source to interact via neutral "photons", let alone form an abelian flux tube. One would therefore expect the adjoint string tension to vanish, rather than Casimir scale. Indeed, if MAQCD is close to CQED, the doubly-charged abelian loop in Eq. (14) is expected to have an area law falloff itself [0,10 and, therefore, for large loops $W_{j=1}^{d} \rightarrow 1 / 3$, and the coresponding potential vanishes. This is verified numerically (Fig. 2 and (7). Thus, the strong version of $A D$ for adjoint sources fails [8.7]. To see whether the weak version can be satisfied we decompose the full adjoint Wilson loop $W_{j=1}=\left(4 w_{0}^{2}-1\right) / 3$ into neutral $(0)$ and charged $( \pm)$ parts

$$
\begin{aligned}
W_{j=1}^{0} & =\frac{2}{3}\left(w_{0}^{2}+w_{3}^{2}\right)-\frac{1}{3} \\
W_{j=1}^{ \pm} & =\frac{2}{3}\left(w_{0}^{2}-w_{3}^{2}\right) .
\end{aligned}
$$

Integrating over $\chi$ as before (see [7] for details)

$$
\begin{aligned}
\left\langle w_{0}^{2}+w_{3}^{2}\right\rangle & =c^{2 L}+s^{2 L}+\sum_{m=1}^{L-1} c^{2 m} s^{2(L-m)}\left(\begin{array}{c}
2 L \\
2 m
\end{array}\right) \\
\Rightarrow\left\langle W_{j=1}^{0}\right\rangle & =\frac{1}{3}\left(1+\langle\cos (2 \phi)\rangle^{2 L}\right)-\frac{1}{3}
\end{aligned}
$$

where $c=\langle\cos \phi\rangle^{2}, s=1-c$. Thus, without offdiagonal gluons $(\cos \phi=1)\left\langle W_{j=1}^{0}\right\rangle \rightarrow 1 / 3$, while with "static" off-diagonal gluons $(\cos \phi<1$, but fixed) one gets $\left\langle W_{j=1}^{0}\right\rangle \rightarrow 0$. However, although 


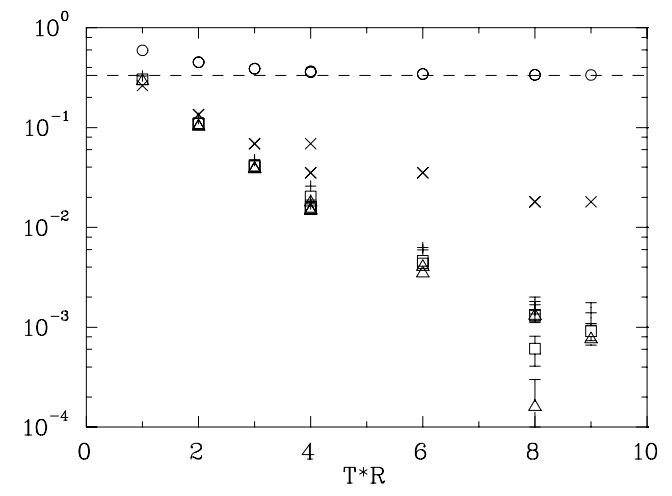

Figure 2. Various adjoint Wilson loops versus loop area: $W_{j=1}(\square), W_{j=1}^{ \pm}(\triangle), W_{j=1}^{0}(+), W_{j=1}^{d}$ (o), Eq. $6(\times)$. Dashed line: the limit $W_{j=1}^{d} \rightarrow \frac{1}{3}$.

this greatly improves Eq. (伍), as seen in Fig. 2, it is not enough for generating an area law for $W_{j=1}^{0}$ : off-diagonal gluons are required dynamically (i.e., $S_{\theta \chi}$ cannot be ignored). On the other hand, for the operator $\left(w_{0}^{2}-w_{3}^{2}\right)$ one finds $[7$

$\left\langle w_{0}^{2}-w_{3}^{2}\right\rangle \approx\langle\cos \phi\rangle^{4 L}\left\langle\cos \left(2 \theta_{L}\right)\right\rangle$.

This is the adjoint analog of Eq. (3); it is seen [7] to be a very good approximation in MA projection (only). A characteristic difference between $\left\langle W_{j=1 / 2}\right\rangle$ and $\left\langle W_{j=1}^{ \pm}\right\rangle$on one hand, and $\left\langle W_{j=1}^{0}\right\rangle$ on the other, is that in the former case the free $\chi$-integration leads to expressions without $\langle\sin \phi\rangle$ terms, whereas in the latter such terms appear, with large degeneracy factors [c.f. Eq. (6)]. Since $S_{\theta \chi}<<S_{\theta}$ relies on ignoring $\sin \phi$ terms, our approximations seem inconsistent in the case of $W_{j=1}^{0}$, which may explain the failure of generating an area law for $W_{j=1}^{0}$ on the basis of these approximations. Numerically (200 measurements on $16^{4}$ and 350 on $12^{4}$ lattices at $\beta=2.4$ ) we find that $W_{j=1}, W_{j=1}^{0}$ and $W_{j=1}^{ \pm}$have within $10 \%$ same Creutz ratios (Fig. 2). This may be explained by using gauge invariance of the energy eigenstates in the spectral decomposition, suggesting that doubly-charged abelian Wilson loops $W_{n=2}$ should be the relevant operators for testing weak abelian dominance in the adjoint case. Evidence in support of this conjecture is shown in Fig. 3. Although encouraging, this result should be treated with caution, unless some better scheme of approximations than the ones suggested in Sec. I succeeds in accounting for the area law behavior of $W_{j=1}^{0}$. Moreover, the pattern of weak AD suggested here should be tested for $j=3 / 2$ sources as well. Work in this direction is in progress.

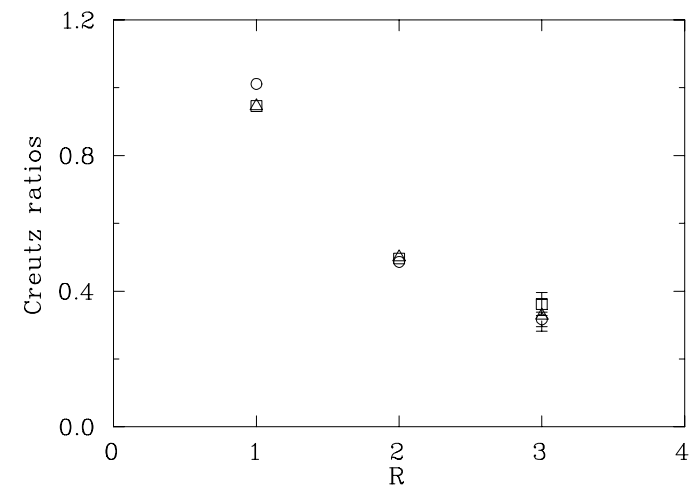

Figure 3. Creutz ratios $\chi[R, R]$ from adjoint Wilson loops $W_{j=1}$ (o) versus ones from doubly charged abelian loops $W_{n=2}$ in MA projection. Results from $16^{4}(\square)$ and $12^{4}(\triangle)$ lattices.

\section{REFERENCES}

1. G. 't Hooft, Nucl. Phys. B190 (1981) 455.

2. A. Kronfeld, M. Laursen, G.Schierholz, U.J. Wiese, Phys. Lett. B198, (1987) 516.

3. T. Suzuki, Nucl. Phys. B (Proc. Suppl.) 30, (1993) 176 ;

4. M. Polikarpov, these proceedings.

5. M. Chernodub, M. Polikarpov and V. Veselov, Phys. Lett. B342 (1995), 303.

6. T. Suzuki and I. Yotsuyanagi, Phys. Rev. D42 (1990) 4257; J.D. Stack, S.D. Neiman and R.J. Wensley, Phys. Rev D50 (1994), 3399.

7. G. Poulis, archive: hep-lat/9601013, to appear in Phys. Rev. D.

8. L. Del Debbio et al., Phys. Rev. D53 (1996) 5891.

9. J. Greensite, these proceedings.

10. G. Bali et al, archive: hep-lat/9603012. 